\title{
Curcumin induces apoptosis by inhibiting BCAT1 expression and mTOR signaling in cytarabine-resistant myeloid leukemia cells
}

\author{
YU-HSIN TSENG ${ }^{1}$, REI-CHENG YANG ${ }^{1-3}$, SHYH-SHIN CHIOU ${ }^{1,2,4,5}$, \\ TZONG-MING SHIEH ${ }^{6,7}$, YIN-HWA SHIH ${ }^{8}$ and PEI-CHIN LIN ${ }^{1,2,4,5}$ \\ ${ }^{1}$ Department of Pediatrics, Kaohsiung Medical University Hospital; ${ }^{2}$ Department of Pediatrics, \\ School of Medicine, College of Medicine; ${ }^{3}$ Graduate Institute of Medicine, \\ College of Medicine; ${ }^{4}$ Division of Hematology and Oncology, Department of Pediatrics; \\ ${ }^{5}$ Special Service Center for Hereditary Hematological Disease, Kaohsiung Medical University Hospital, \\ Kaohsiung Medical University, Kaohsiung 80756; ${ }^{6}$ School of Dentistry, College of Dentistry; \\ ${ }^{7}$ Department of Dental Hygiene, College of Health Care, China Medical University, Taichung 40402; \\ ${ }^{8}$ Department of Healthcare Administration, Asia University, Taichung 41354, Taiwan, R.O.C.
}

Received December 15, 2020; Accepted May 11, 2021

DOI: $10.3892 / \mathrm{mmr} .2021 .12204$

\begin{abstract}
Cytarabine is a key chemotherapy drug for treating leukemia; however, chemotherapy-induced multidrug resistance is a major cause of therapy failure or tumor recurrence. Current medical treatment strategies still cannot address the issue of multidrug resistance phenotypes in the treatment of leukemia. Curcumin counteracts tumor development by inducing apoptosis in cytarabine-resistant acute myeloid leukemia cells. Branched-chain amino acid transaminase 1 (BCAT1), an aminotransferase enzyme, acts on branched-chain amino acids. Moreover, the aberrant expression of BCAT1 has been observed in numerous cancer cells, and BCAT1 serves a critical role in the progression of myeloid leukemia. BCAT1 can interfere with cancer cell proliferation by regulating mTOR-mediated mitochondrial biogenesis and function. The present study aimed to investigate whether curcumin induces apoptosis by regulating BCAT1 expression and mTOR signaling in cytarabine-resistant myeloid leukemia cells. Four leukemia cell lines and three primary myeloid leukemia cells were treated with curcumin, and the expression and activity of BCAT1 and mTOR were investigated by reverse transcription-quantitative PCR, western blotting and $\alpha-K G$ quantification assay. The results demonstrated that curcumin inhibited BCAT1 expression in Kasumi-1, KG-1, HL60, cytarabine-resistant HL60, and cytarabine-resistant primary myeloid leukemia cells. Notably, tetrahydrocurcumin, a major metabolite of curcumin,
\end{abstract}

\footnotetext{
Correspondence to: Dr Pei-Chin Lin, Division of Hematology and Oncology, Department of Pediatrics, Kaohsiung Medical University Hospital, Kaohsiung Medical University, 100 Tzyou 1st Road, Kaohsiung 80756, Taiwan, R.O.C.

E-mail: cooleylin@gmail.com
}

Key words: curcumin, branched-chain amino-acid transaminase 1, mTOR, myeloid leukemia, cytarabine, apoptosis and cytarabine had no inhibitory effect on BCAT1 expression. Furthermore, BCAT1 and mTOR signaling may modulate each other in cytarabine-resistant HL60 cells. The present results indicated that curcumin may induce apoptosis by inhibiting the BCAT1 and mTOR pathways. Thus, understanding the mechanism underlying curcumin-induced apoptosis in cytarabine-resistant cells can support the development of novel drugs for leukemia.

\section{Introduction}

Leukemia is a heterogeneous group of hematological cancer types and is the most common type of childhood malignancy, accounting for $\leq 30 \%$ of all childhood malignancies (1). Leukemia is classified into four types: Acute myeloid leukemia (AML), chronic myeloid leukemia (CML), acute lymphoblastic leukemia and chronic lymphocytic leukemia (2). Cytarabine is a key chemotherapy drug for leukemia treatment. However, preventing the side effects of chemotherapy drugs and their ability to induce multidrug resistance phenotypes remains challenging (3). Emerging cancer treatment strategies focus on reducing drug toxicity and multidrug resistance phenotypes (4). Curcumin is a yellow spice and phenolic compound derived from the plant Curcuma longa, and previous studies have reported that it is a natural phytochemical with the potential to overcome drug resistance (5-7). Another study also observed curcumin induced apoptosis in cytarabine-resistant HL60 cells (4).

Branched-chain amino acids (BCAAs) are essential amino acids (8). For example, to achieve rapid proliferation, cancer cells must obtain BCAAs via the circulation or from surrounding tissues (9). A retrospective metabolomic study reported that elevated plasma BCAA levels were associated with a $>2$ fold increased risk of pancreatic cancer (10). In addition, amino acid levels in hepatocellular carcinoma, gastric cancer and colon cancer tissues are typically higher compared with those in the respective non-tumorous tissues (11). 
Reprogrammed cellular metabolism is a common characteristic of various cancer types (12-14). However, whether metabolic changes directly regulate cancer development and progression remains poorly understood.

BCAA transaminase 1 (BCAT1) is a cytosolic aminotransferase for BCAAs and is aberrantly activated in several types of cancer, including esophageal squamous cell carcinoma (15), gastric cancer (16), breast cancer (17), hepatocellular carcinoma (18) and myeloid leukemia (19). BCAT1 is upregulated during the progression of CML and promotes BCAA production in leukemia cells via the amination of branched-chain keto acids. Furthermore, blocking the expression or activity of BCAT1 can induce cell differentiation and impair the propagation of blast crisis CML (19). Another study indicated that AML with high levels of BCAT1 exhibited a DNA hypermethylation phenotype similar to cases carrying a mutant isocitrate dehydrogenase (IDHmut), in which the ten-eleven translocation-2 (TET2) protein is inhibited by the oncometabolite 2-hydroxyglutarate (20). Moreover, high levels of BCAT1 are closely associated with shorter overall survival in IDH wild-type (wt)/TET2wt but not in IDHmut or TET2mut AML (20).

It has been shown that BCAT1 is a key regulator of intracellular $\alpha$-ketoglutarate $(\alpha-K G)$ levels in various types of tumor cells, and changes in intracellular $\alpha-\mathrm{KG}$ levels have a major effect on AML cell biology $(20,21)$. BCAT1 knockdown in leukemia cells causes $\alpha-K G$ accumulation, resulting in EGL-9 family hypoxia inducible factor 1-mediated hypoxia-inducible factor- $1 \alpha$ protein degradation (20). This results in defects in growth and survival of leukemia cell lines, as well as the abrogation of leukemia-initiating potential. By contrast, BCAT1 overexpression in leukemia cells reduces intracellular $\alpha-K G$ levels and leads to DNA hypermethylation by altering TET activity (20). However, this transamination reaction is reported to be reversible. BCAT1 catalyzes the transamination of plasma BCKAs to generate BCAAs in order to maintain nutrient sensing via the mTOR complex 1 (mTORC1), thereby maintaining proliferation signals in leukemia cells (22).

BCAAs also have crucial allosteric regulation and signal transduction effects. Among these effects, leucine-induced mTOR pathway regulation is the most widely discussed. It has been shown that BCAT1 blockade significantly reduced mTORC1 activity in K562 and MCF-7 cells $(17,19)$. Moreover, cell proliferation and colony formation assays revealed that rapamycin neutralizes the promotive effects of BCAT1 on the cell proliferation rate and colony formation capacity of cancer cells, suggesting that mTOR activity contributes to BCAT1 function in tumor progression $(17,23)$. The mTOR pathway is the catalytic subunit of two distinct multiprotein complexes, namely mTORC1 and mTORC2, and it is a critical integrator of growth factor-activating and nutrient-sensing pathways to modulate various cell functions, including survival, proliferation, differentiation, autophagy, apoptosis and metabolism $(24,25)$. Moreover, up to $80 \%$ of human cancer types involve mTORC1 signal dysregulation (26).

Previous studies have reported that curcumin can regulate several molecules in cell signal transduction pathways, including mTOR $(27,28)$. The anticancer effects of curcumin are reflected in its ability to induce growth arrest and apoptosis in various premalignant and malignant cells (29). However, to the best of the authors' knowledge, studies have not investigated the regulatory effect of curcumin on BCAT1. To fill this gap in the literature, the present study investigated whether curcumin induces apoptosis by regulating mTOR and BCAT1 signaling in cytarabine-resistant myeloid cells.

\section{Materials and methods}

Cell culture and drug treatment. Kasumi-1, KG-1 and HL60 are three common myeloid leukemia cell lines and were purchased from ATCC. Kasumi-1 cells were cultured with RPMI 1640 medium (cat. no. A1049101; Gibco; Thermo Fisher Scientific, Inc.) with $20 \%$ FBS (Thermo Fisher Scientific, Inc.). KG-1 cells were cultured with Iscove's modified Dulbecco's medium (cat. no. SH30228.02; HyClone; Cytiva) with 20\% FBS. HL60 and resistant (R)-HL60 cells were cultured with RPMI 1640 medium supplemented with 10\% FBS. The R-HL60 cell line, which was established in the Yu-Hsin Tseng's laboratory (Department of Pediatrics, Kaohsiung Medical University Hospital), had a cytarabine resistance level $>1,000$ times that of the parent HL60 cells (4). All cells are cultured at an incubator with $5 \% \mathrm{CO}_{2}$ and temperature of $37^{\circ} \mathrm{C}$.

Curcumin, tetrahydrocurcumin, cytarabine and PP242 were purchased from Sigma-Aldrich (Merck KGaA). The BCATc inhibitor 2 was purchased from Cayman Chemical Company. Stock solutions of curcumin $(50 \mathrm{mM})$, tetrahydrocurcumin $(100 \mathrm{mM}), \mathrm{PP} 242(10 \mathrm{mM})$ and BCATc inhibitor 2 (80 $\mathrm{mM})$ were dissolved in DMSO, and stock solution of cytarabine $(400 \mathrm{mM})$ was dissolved in $\mathrm{ddH}_{2} \mathrm{O}$. The working concentration and durations of drug treatment were $0-50 \mu \mathrm{M}$ curcumin for 2-48 h, 0-100 $\mu \mathrm{M}$ tetrahydrocurcumin for $24 \mathrm{~h}$, $0-400 \mu \mathrm{M}$ cytarabine for $24 \mathrm{~h}, 10 \mu \mathrm{M}$ PP242 for $24 \mathrm{~h}$ and $80 \mu \mathrm{M}$ BCATc inhibitor 2 for $24-48 \mathrm{~h}$. The process of drug treatment was performed in a $37^{\circ} \mathrm{C}$ incubator with $5 \% \mathrm{CO}_{2}$.

Patients and samples. Patients were recruited from the inpatients department at Kaohsiung Medical University Hospital between December 2017 and December 2018. The present study was approved by the Institutional Review Board of Kaohsiung Medical University Hospital [approval no. KMUHIRB-SV(I)-20170038]. Written informed consents of patients were obtained from each participant. Bone marrow samples were obtained from three patients with relapsed cytarabine-resistant myeloid leukemia. The characteristic gene alterations of three patients included FLT3 internal tandem duplication (FLT3-ITD) and nucleophosmin 1cooperating mutations, Breakpoint Cluster Region Protein (BCR)-Abelson Tyrosine-Protein Kinase 1 (ABL) mutations (BCR and ABL genes break off and switch places to form a fusion protein) and FLT3-ITD mutations. Patients were 9-year-old male, 17-year-old female and 7-year-old male. The mononuclear cells were isolated by centrifuging at $1,200 \mathrm{x} \mathrm{g}$ for $20 \mathrm{~min}$ at room temperature using the Ficoll-Paque method (GE Healthcare) (30). The percentage of malignant blasts in bone marrow was the diagnostic basis and treatment outcome assessment for patients with AML (31). Complete remission, partial remission and relapse disease were defined as $<5,5-20$ and $>20 \%$ malignant blasts in bone marrow, respectively. The percentages of malignant blasts in 
A

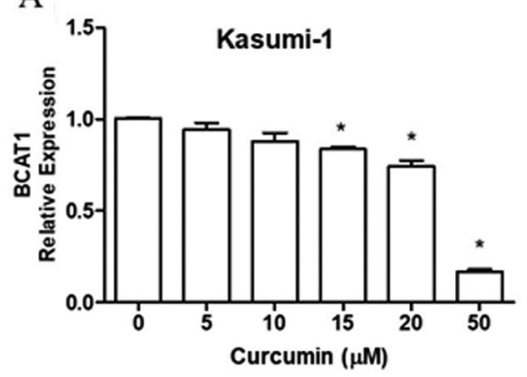

B

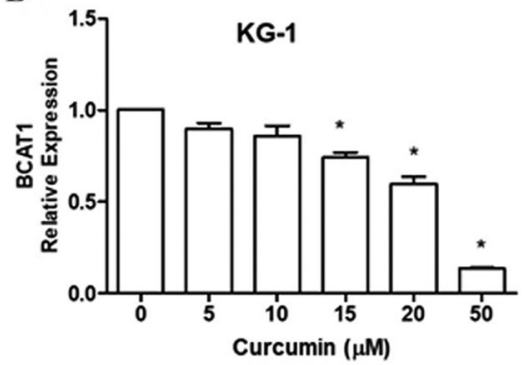

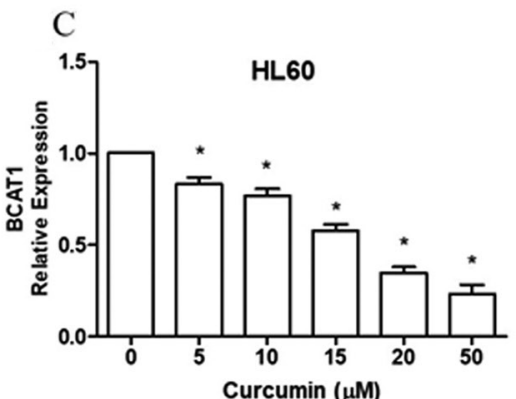

Figure 1. Curcumin inhibits the expression level of BCAT1 mRNA in myeloid cell lines. (A) Kasumi-1, (B) KG-1 and (C) HL60 cells were treated with 0-50 $\mu \mathrm{M}$ curcumin for $24 \mathrm{~h}$, and the expression level of BCAT1 mRNA was detected using reverse transcription-quantitative PCR. Curcumin reduced BCAT1 mRNA expression in all three cell lines. The data represent the mean \pm SEM of three independent experiments with triple replicates and were analyzed using the one-way ANOVA test to determine the differences of multiple groups. Bonferroni was used as a post hoc test. "P<0.05 the experimental group vs. the vehicle control group. BCAT1, branched-chain amino acid transaminase 1.

the three bone marrow samples collected in this study were $48.6,21.1$ and $22.1 \%$, respectively (data collected from clinical medical records).

RNA isolation and reverse transcription-quantitative PCR $(R T-q P C R)$. Total RNA was isolated using a TRIzol ${ }^{\circledR}$ total RNA extraction kit (Thermo Fisher Scientific, Inc.), and cDNA was synthesized using a Maxima First Strand cDNA Synthesis kit (Thermo Fisher Scientific, Inc.). The reaction steps of RT were: Incubation $10 \mathrm{~min}$ at $25^{\circ} \mathrm{C}$, followed by $15 \mathrm{~min}$ at $50^{\circ} \mathrm{C}$, and termination of the reaction by heating $5 \mathrm{~min}$ at $85^{\circ} \mathrm{C}$. Amplification reactions of qPCR was set up in $10 \mu \mathrm{l}$ reaction volumes containing amplification primers and Fast SYBR Green Master mix (Thermo Fisher Scientific, Inc.) and detected by an ABI 7500 system (Applied Biosystems; Thermo Fisher Scientific, Inc.). Initial denaturation temperature was increased to $95^{\circ} \mathrm{C}$ for $10 \mathrm{~min}$, following by 40 cycles of denaturation at $95^{\circ} \mathrm{C}$ for $15 \mathrm{sec}$, annealing at $60^{\circ} \mathrm{C}$ for $60 \mathrm{sec}$ and extension at $72^{\circ} \mathrm{C}$ for $15 \mathrm{sec}$. GAPDH expression was used as the internal control and was quantified using the $2^{-\Delta \Delta \mathrm{Cq}}$ method (32). The primer sequences were as follows: BCAT1 forward, 5'-TTC AACTCGTGATACACCAA-3' and reverse, 5'-ATTCCTGTG CTAGAGAGCAT-3'; and GAPDH forward, 5'-CTGGGCTAC ACTGAGCACC-3' and reverse, 5'-AAGTGGTCGTTGAGG GCAATG-3'.

Western blotting. R-HL60 cells were lysed using RIPA lysis buffer with the protease inhibitor and phosphatase inhibitor (Thermo Fisher Scientific, Inc.). The samples were centrifuged at $13,000 \times \mathrm{g}$ for $15 \mathrm{~min}$ at $4^{\circ} \mathrm{C}$, and the supernatant proteins were then collected for western blotting. The protein concentrations were determined using a Pierce BCA protein assay kit (Thermo Fisher Scientific, Inc.). Protein (20 $\mu \mathrm{g}$ per well) was loaded on $4-12 \%$ Bolt Bis-Tris Plus gels (Invitrogen; Thermo Fisher Scientific, Inc.) and transferred to PVDF membranes. The membranes were blocked with 5\% BSA (Sigma-Aldrich; Merck KGaA) containing $0.1 \%$ Tween-20 for $1 \mathrm{~h}$ at room temperature and then incubated overnight with primary antibodies against human phosphorylated (p)-mTOR (ser2448; 1:1,000; Arigo Biolaboratories; cat. no. ARG40666), total (t)-mTOR (1:1,000; Arigo Biolaboratories; cat. no. ARG57640), BCAT1 (1:3,000; Cell Signaling Technology, Inc.; cat. no. 12822), poly (ADP-ribose) polymerase 1 (PARP 1; 1:2,000; Abcam; cat. no. ab32138), cleaved (c)-PARP 1 (1:15,000; Abcam; cat. no. ab32064) and GAPDH (1:30,000; Ambion; Thermo Fisher Scientific, Inc.; cat. no. AM4300) at $4^{\circ} \mathrm{C}$. The primary antibodies were washed in phosphate-buffered saline plus $0.1 \%$ Tween-20, then the blots were incubated with HRP-linked secondary antibodies (1:10,000; Cytiva; cat. no. NA9310) for $1 \mathrm{~h}$ at room temperature. Bands were visualized using an ECL assay kit (Thermo Fisher Scientific, Inc.). X-ray film was used for chemiluminescence detection. The densitometry of quantify protein bands from western blot films were analyzed using ImageJ $1.52 \mathrm{t}$ software (National Institutes of Health).

$\alpha-K G$ quantification assay. In total, $2 \times 10^{6} \mathrm{R}-\mathrm{HL} 60$ cells were harvested for each assay. Cells were washed with cold PBS and lysed with $100 \mu \mathrm{l}$ lysis buffer. The deproteinization step was performed using a ReadiUs TCA Deproteinization Sample Preparation kit (AAT Bioquest, Inc.). Subsequently, $\alpha$-KG levels were determined using the $\alpha-K G$ quantitation assay kit (AAT Bioquest, Inc.), according to the manufacturer's instructions.

Statistical analysis. Statistical analyses were performed using GraphPad Prism 5.0 (GraphPad Software, Inc.). The paired t-tests and one-way ANOVA were used to determine the differences between the experimental and control groups and Bonferroni was used as a post hoc test following ANOVA. Error bars presented herein represent the mean \pm SEM from triple replicates. $\mathrm{P}<0.05$ was considered to indicate a statistically significant difference in all comparisons of the experimental group with the vehicle control group $\left(\mathrm{H}_{2} \mathrm{O}\right.$ for cytarabine, DMSO for curcumin, tetrahydrocurcumin, PP242 and BCATc inhibitor 2).

\section{Results}

Curcumin inhibits the mRNA expression levels of BCAT1 in myeloid cell lines. The human myeloid leukemia cell lines Kasumi-1 (Fig. 1A), KG-1 (Fig. 1B) and HL60 (Fig. 1C) were treated with different concentrations of curcumin for $24 \mathrm{~h}$. The results demonstrated that curcumin effectively reduced the mRNA expression levels of BCAT1 in a dose-dependent manner.

Curcumin inhibits the MRNA expression levels of BCATI in cytarabine-resistant myeloid leukemia cells. R-HL60, a 
A

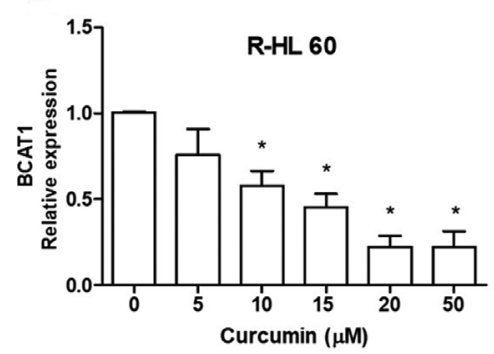

$\mathrm{C}$

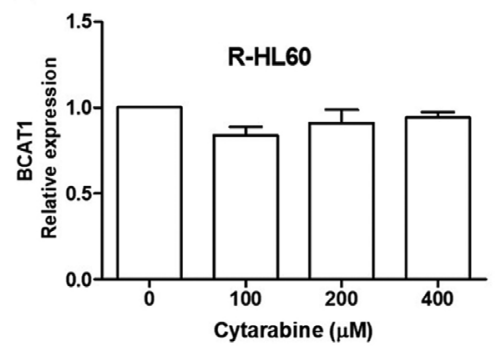

$\mathrm{B}$

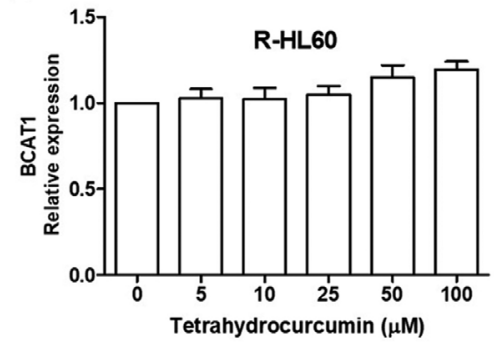

$\mathrm{D}$



Figure 2. Curcumin inhibits the expression level of BCAT1 mRNA in cytarabine-resistant myeloid leukemia cells. R-HL60 cells were treated with (A) 0-50 $\mu \mathrm{M}$ curcumin, (B) 0-100 $\mu \mathrm{M}$ tetrahydrocurcumin and (C) 0-400 $\mu \mathrm{M}$ cytarabine for $24 \mathrm{~h}$, and the expression level of BCAT1 mRNA was detected using RT-qPCR. Curcumin, but not tetrahydrocurcumin and cytarabine reduced BCAT1 mRNA expression. (D) Monocytes were isolated from bone marrow samples collected from patients with cytarabine-resistant acute myeloid leukemia and were treated with $0-20 \mu \mathrm{M}$ curcumin for $24 \mathrm{~h}$. The expression level of BCAT1 mRNA was detected using RT-qPCR, and the results revealed that curcumin decreased BCAT1 mRNA expression. The data represented the mean \pm SEM of three independent experiments with triple replicates per experiment and were analyzed using one-way ANOVA to determine the differences of multiple groups. Bonferroni was used as a post hoc test. "P $<0.05$ the experimental group vs. the vehicle control group. BCAT1, branched-chain amino acid transaminase 1; R-, resistant; RT-qPCR, reverse transcription-quantitative PCR.

A

\begin{tabular}{|c|c|c|c|c|c|c|}
\hline \multicolumn{6}{|c|}{24 hours } & \multirow{3}{*}{$\begin{array}{l}\text { Curcumin }(\mu M \\
\text { p-mTOR }\end{array}$} \\
\hline $\mathbf{0}$ & 5 & 10 & 15 & 20 & 50 & \\
\hline$=$ & $=$ & $=$ & $=$ & - & $7=$ & \\
\hline & $=$ & $=$ & $=$ & 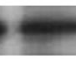 & -6 & t-mTOR \\
\hline$=$ & $=$ & - & - & $=$ & - & BCAT1 \\
\hline & & & & & & \\
\hline
\end{tabular}

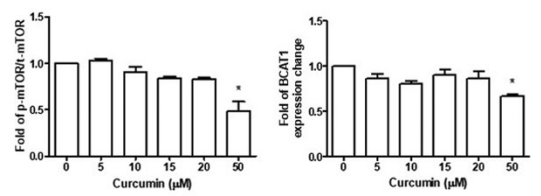

B
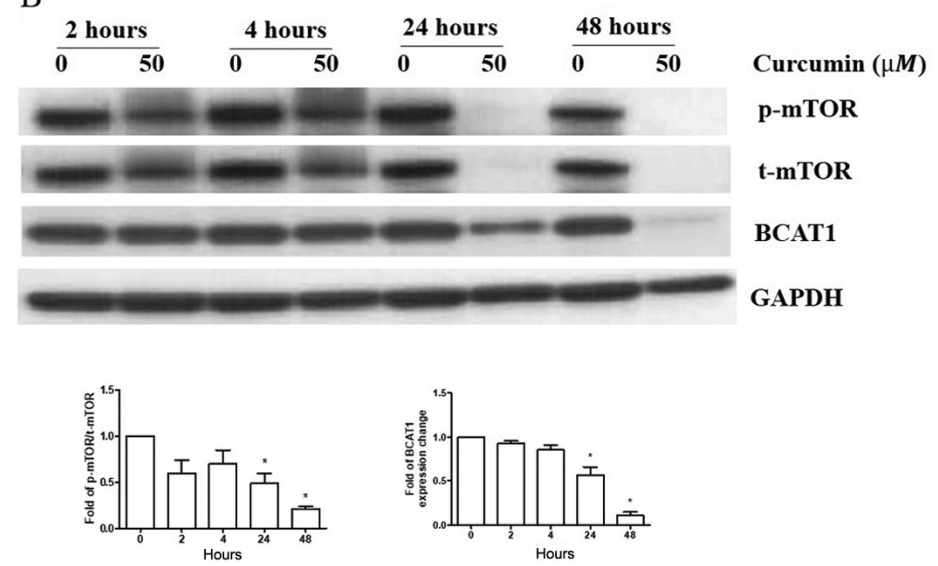

Figure 3. Curcumin inhibits BCAT1 expression and mTOR signaling in R-HL60 cells. (A) R-HL60 cells were treated with 0-50 $\mu \mathrm{M}$ curcumin for 24 h. (B) R-HL60 cells were treated with curcumin at a concentration of $50 \mu \mathrm{M}$ for $2-48 \mathrm{~h}$. Curcumin caused the reduction of the ratio of p-mTOR/t-mTOR and BCAT1 protein expression levels. The data represent the mean \pm SEM of three independent experiments with triple replicates and were analyzed using the one-way ANOVA test to determine the differences of multiple groups. Bonferroni was used as a post hoc test. "P<0.05 the experimental group vs. the vehicle control group. BCAT1, branched-chain amino acid transaminase 1; R-, resistant; p-, phosphorylated; t-, total.

cytarabine-resistant HL60 cell line, was treated with 0-50 $\mu \mathrm{M}$ curcumin, 0-100 $\mu \mathrm{M}$ tetrahydrocurcumin and 0-400 $\mu \mathrm{M}$ cytarabine for $24 \mathrm{~h}$. The results indicated that curcumin (Fig. 2A), but not tetrahydrocurcumin (Fig. 2B) or cytarabine (Fig. 2C), effectively decreased the mRNA expression levels of BCAT1. The cytotoxicity of cytarabine in mononuclear cells isolated from the bone marrow of three patient-derived with AML was measured using an XTT assay. The $\mathrm{IC}_{50}$ values of cytarabine were 169, 749 and $>1,600 \mu \mathrm{M}$, respectively (data not shown).
Curcumin also reduced the mRNA expression levels of BCAT1 in these mononuclear cells (Fig. 2D).

Curcumin inhibits BCAT1 protein expression and $m$ TOR signaling in R-HL60 cells. R-HL60 cells were treated with $0-50 \mu \mathrm{M}$ curcumin for $24 \mathrm{~h}$. The results indicated that only $50 \mu \mathrm{M}$ curcumin treatment effectively reduced the ratio of $\mathrm{p}-\mathrm{mTOR} / \mathrm{t}-\mathrm{mTOR}$ and $\mathrm{BCAT} 1$ protein expression compared with the vehicle control group. (Fig. 3A), so this 
A
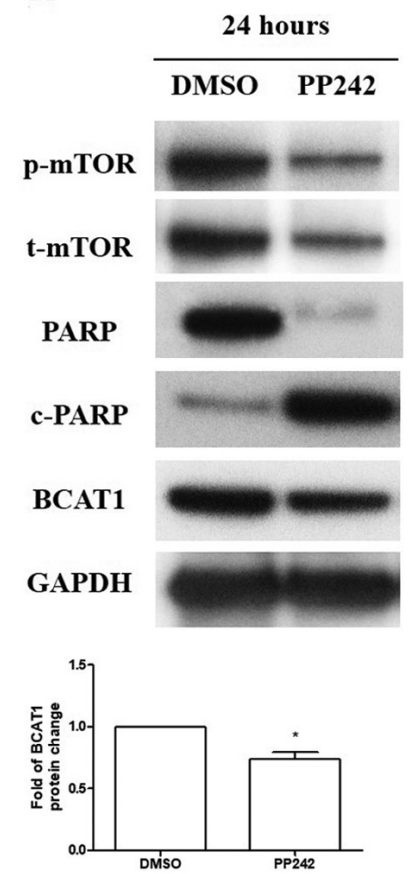
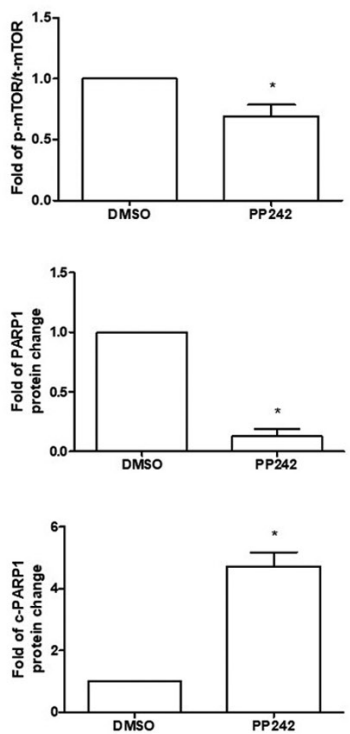

B

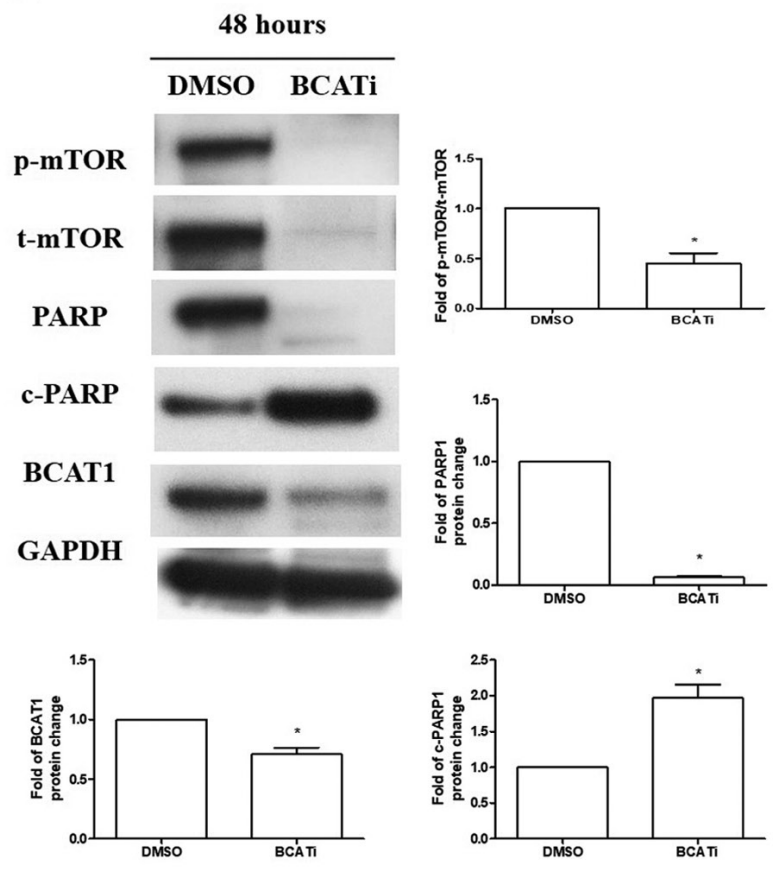

Figure 4. mTOR and BCAT1 pathways can modulate each other and regulate apoptosis. R-HL60 cells were treated with (A) $10 \mu \mathrm{M}$ PP242 for $24 \mathrm{~h}$ or (B) $80 \mu \mathrm{M}$ BCATc inhibitor 2 for $48 \mathrm{~h}$, and p-mTOR, t-mTOR, PARP1, c-PARP1 and BCAT1 protein expression levels were detected using western blotting. Both PP242 and BCATc inhibitor 2 significantly decreased the ratio of p-mTOR/t-mTOR, PARP1 and BCAT1 expression and significantly induced c-PARP1 expression. The data were compared with the vehicle control group (DMSO) and represented the mean \pm SEM of three independent experiments with triple replicates per experiment. Paired t-tests was used to determine the differences between the experimental and control groups. "P<0.05 the experimental group vs. the vehicle control group. BCATi, BCATc inhibitor 2; BCAT, branched-chain amino acid transaminase; R-, resistant; p-, phosphorylated; t-, total; c-, cleaved; PARP 1, poly (ADP-ribose) polymerase 1 .

concentration was chosen to treat cells for different time periods. The results indicated that curcumin reduced the ratio of $\mathrm{p}-\mathrm{mTOR} / \mathrm{t}-\mathrm{mTOR}$ and BCAT1 protein expression in 24-48 h (Fig. 3B).

BCAT1 and mTOR pathways modulate each other and regulate apoptosis in $\mathrm{R}-\mathrm{HL} 60$ cells. R-HL60 cells were treated with $10 \mu \mathrm{M}$ PP242 for $24 \mathrm{~h}$ or $80 \mu \mathrm{M}$ BCATc inhibitor 2 for $48 \mathrm{~h}$ to inhibit the mTOR or BCAT1 pathway, respectively. The results demonstrated that the inhibition of mTOR signaling by PP242 reduced the expression levels of the BCAT1 and PARP1 proteins, and induced c-PARP1 protein expression (Fig. 4A). Moreover, inhibition of BCAT1 signaling through use of the BCATc inhibitor 2 decreased the ratio of $\mathrm{p}-\mathrm{mTOR} / \mathrm{mTOR}$ and PARP1 protein expression levels and induced c-PARP1 protein expression (Fig. 4B).

Curcumin, PP242 and BCATc inhibitor 2 inhibit $\alpha-K G$ levels in R-HL60 cells. R-HL60 cells were treated with $50 \mu \mathrm{M}$ curcumin, $10 \mu \mathrm{M}$ PP2 24 or $80 \mu \mathrm{M}$ BCATc inhibitor 2 for $24 \mathrm{~h}$. The results indicated that curcumin, PP242 and BCATc inhibitor 2 effectively decreased the levels of $\alpha-\mathrm{KG}$ (Fig. 5).

A schematic diagram of curcumin regulating cell apoptosis through the BCAT1 and mTOR pathways. A schematic of the possible mechanism via which curcumin regulates apoptosis by regulating the BCAT1 and mTOR pathways in R-HL60 cells is given as Fig. 6 .

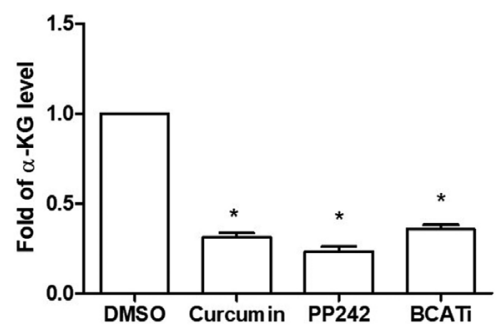

Figure 5. Curcumin, PP242 and BCATi inhibit $\alpha$-KG levels in R-HL60 cells. R-HL60 cells were treated with $50 \mu \mathrm{M}$ curcumin, $10 \mu \mathrm{M}$ PP242 or $80 \mu \mathrm{M}$ BCATi for $24 \mathrm{~h}$. Subsequently, $\alpha$-KG levels were detected using an $\alpha-K G$ quantitation assay kit. Curcumin, PP242 and BCATi significantly decreased $\alpha-K G$ levels. The data represented the mean \pm SEM of three independent experiments with triple replicates and were analyzed using the one-way ANOVA test to determine the differences of multiple groups. Bonferroni was used as a post hoc test. Curcumin, PP242 and BCATi treatment decreased $\alpha$-KG level in the R-HL60 cells. ${ }^{*} \mathrm{P}<0.05$ the experimental group vs. the vehicle control group. BCATi, BCATc inhibitor 2; BCAT, branched-chain amino acid transaminase; $\mathrm{R}-$, resistant; $\alpha$-KG, $\alpha$-ketoglutarate.

\section{Discussion}

Resistance to chemotherapy is a major reason for treatment failure. Our previous study reported that curcumin can induce the apoptosis in R-HL60 cells (4). The present study further investigated the mechanism of apoptosis, which may be helpful for the treatment of cytarabine-resistant leukemia. The current results demonstrated that curcumin induced apoptosis by inhibiting the BCAT1 and mTOR pathways, and the two pathways exhibited crosstalk in R-HL60 cells. 


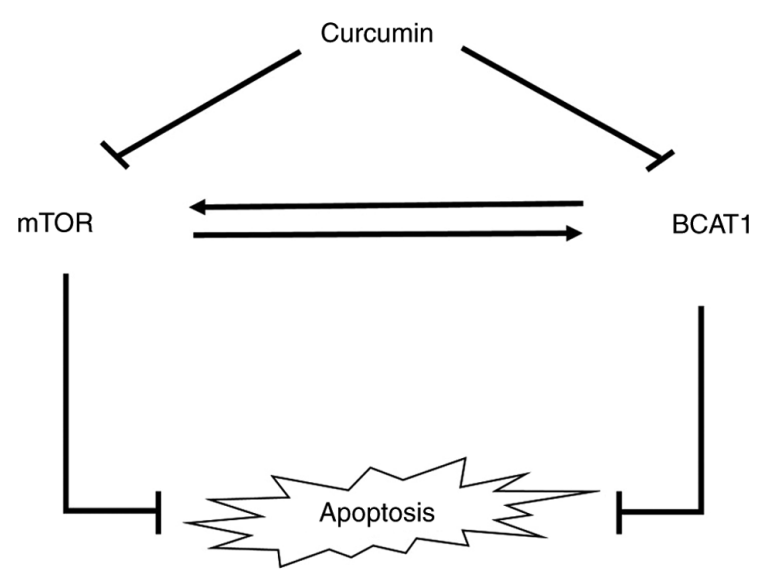

Figure 6. Schematic of curcumin regulating apoptosis via the BCAT1 and mTOR pathways. BCAT1, branched-chain amino acid transaminase 1 .

Previous studies have revealed that high levels of BCAT1 are closely associated with shorter overall survival in IDHwtTET2wt but not in IDHmut or TET2mut AML (20,21). The patients recruited in the current study are AML with IDHwtTET2wt, so BCAT1 activation served a key role in the development of these tumors. The results of the current study indicated that curcumin treatment reduced the expression level of BCAT1, which suggested that curcumin can interfere with the development of cancer cells through BCAT1 signaling. To the best of the authors' knowledge, the present study was the first to investigate the mechanism underlying curcumin's apoptotic effects by inhibiting BCAT1 expression. Tetrahydrocurcumin is a major curcumin metabolite, and thus, it was also examined whether tetrahydrocurcumin regulates BCAT1 expression. Curcumin and tetrahydrocurcumin are known to induce cytarabine-resistant HL60 cell death via distinct pathways (apoptosis and autophagy, respectively) (4). Therefore, the current results demonstrating that tetrahydrocurcumin differed from curcumin in its effect on BCAT1 expression were reasonable. In addition, R-HL60 cells are resistant to cytarabine treatment, and therefore, cytarabine treatment can be used as a negative control group for curcumin treatment to induce apoptosis via the mTOR and BCAT1 pathways.

Previous studies have reported that abnormal expression or functional activity in mTOR leads to the occurrence, progression and drug resistance of various types of tumors $(33,34)$. Moreover, curcumin acts as an antitumor agent that inhibits various signaling pathways, especially mTOR (6). Studies have also revealed that curcumin inhibits the proliferation of cancer cells via PARP1 cleavage $(35,36)$. PARP1 is a nuclear enzyme, and its upregulation has been observed in various primary human cancer cell lines. PARP1 is cleaved into fragments during apoptosis, and c-PARP1 has become a useful marker of apoptosis (6). The ability of mTOR and BCAT1 to regulate cell apoptosis has been previously reported $(37,38)$; however, to the best of the authors' knowledge, the present study was the first to indicate that curcumin can inhibit BCAT1 protein expression.

BCAT1 blockade reduces mTORC1 activity; however, to the best of the authors' knowledge, this is the first report indicating that the mTOR and BCAT1 pathways can modulate each other. Drugs targeting mTORC1 have been used to treat various types of malignant tumors; however, the feedback caused by long-term inhibition of mTOR results in the necessity of additional cycles to compensate for factors that promote survival $(37,39)$. Previous studies have highlighted that by targeting the downstream components of mTOR, the problems associated with the feedback mechanism can be bypassed, thereby avoiding efficiency limitations, limiting the toxicity caused by complete mTOR blockade and avoiding the targeting of other kinases $(25,40)$. This indicates that curcumin may have a stronger apoptotic effect on R-HL60 cells than mTOR inhibitors by blocking both the BCAT1 and mTOR pathways.

Studies have reported that histone modification was critical for the activation of BCAT1. For example, histone H3 lysine 9 (H3K9) demethylation promotes BCAT1 upregulation, thereby inducing tyrosine kinase inhibitor resistance-mediated BCAT1 activation in lung cancer cells (41). Curcumin is known to increase histone deacetylase 2 expression, reduced the levels of $\mathrm{H} 3 / \mathrm{H} 4$ acetylation and increased H3K9 methylation in the promoter region of IL-8, monocyte chemoattractant protein-1 and macrophage inflammatory protein $2 \alpha$ genes (42). Moreover, an in vitro study revealed that curcumin inhibited the acetylation of $\mathrm{H} 3 \mathrm{~K} 9$, as well as reversed the upregulation of caspase activity and downregulation of Bcl-2 in alcohol-induced apoptosis in cardiac cells (43). Although, to the best of the authors' knowledge, no research has yet investigated the regulatory effect of curcumin on BCAT1, it can be suggested that curcumin may inhibit BCAT1 activation by regulating the methylation and/or acetylation of H3K9. In addition, mTOR can regulate histone methylation and demethylation. For example, mTORC1 phosphorylates the H3K9 demethylase jumonji domain containing 1C (JMJD1C) in a nutrient-dependent manner. The p-JMJD1C then interacts with the transcription factor USF-1 to demethylate H3K9me2 at genes promoting lipogenesis in the liver $(44,45)$. However, whether mTOR induces BCAT1 expression by demethylating H3K9me2 and whether curcumin inhibits the expression level of BCAT1 by regulating methylation and acetylation of $\mathrm{H} 3 \mathrm{~K} 9$ require further investigation.

Although BCAT1 activity reportedly restricts $\alpha-\mathrm{KG}$ levels, this transamination reaction is also considered reversible. BCAT1 catalyzes the transamination of plasma BCKAs to generate BCAAs, and BCAT1 maintains nutrient sensing via mTORC1 to sustain proliferative signaling in leukemia cells (20). Moreover, BCAAs or $\alpha-K G$ supplementation reverse the colony forming ability of BCAT1 knockdown cells (19). The present results indicated that curcumin, PP242 and BCATc inhibitor 2 significantly reduced $\alpha-K G$ levels. This finding suggests that curcumin reduces the level of $\alpha-\mathrm{KG}$ by inhibiting the BCAT1 and mTOR pathways, ultimately leading to cancer cell death.

The collection of clinical samples must be coordinated with the immediate cell culture tests in the laboratory, so it is relatively difficult to obtain data. How to collect more experimental data of clinical samples and to study whether curcumin inhibits the expression level of BCAT1 by regulating histone methylation and acetylation requires further investigations. In conclusion, curcumin regulates the mTOR pathway; however, the present study, to the best of the authors' 
knowledge, revealed for the first time that curcumin inhibited BCAT1 expression and cell apoptosis via the simultaneous regulation of the mTOR and BCAT1 pathways. This discovery may broaden the possible treatment options for leukemia.

\section{Acknowledgements}

Not applicable.

\section{Funding}

This study was supported by a grant from the Ministry of Scienceand Technology (grantnos.MOST108-2635-B-037-005 and MOST109-2314-B-037-103-MY3) and Kaohsiung Medical University Hospital (grant nos. KMUH106-M620 and KMUH108-8R45).

\section{Availability of data and materials}

The datasets used and/or analyzed during the current study are available from the corresponding author on reasonable request.

\section{Authors' contributions}

YHT, RCY, SSC, TMS, YHS and PCL made substantial contributions to conception and design, or acquisition of data, or analysis and interpretation of data, and confirm the authenticity of all the raw data. YHT, SSC, TMS and YHS been involved in drafting the manuscript or revising it critically for important intellectual content. All authors have read and approved the final version of the manuscript.

\section{Ethics approval and consent to participate}

This study was approved by the Institutional Review Board of Kaohsiung Medical University Hospital [approval no. KMUHIRB-SV(I)-20170038]. Written informed consents of patients were obtained from each participant between 2017-2018.

\section{Patient consent for publication}

Not applicable.

\section{Competing interests}

The authors declare that they have no competing interests.

\section{References}

1. Madhusoodhan PP, Carroll WL and Bhatla T: Progress and prospects in pediatric leukemia. Curr Probl Pediatr Adolesc Health Care 46: 229-241, 2016.

2. Lyengar V and Shimanovsky A: Leukemia. In: StatPearls StatPearls Publishing Copyright (C) 2021, StatPearls Publishing LLC., Treasure Island, FL, 2021.

3. Liu N, Wang C, Wang L, Gao L, Cheng H, Tang G, Hu X and Wang J: Valproic acid enhances the antileukemic effect of cytarabine by triggering cell apoptosis. Int J Mol Med 37: 1686-1696, 2016.

4. Tseng YH, Chiou SS, Weng JP and Lin PC: Curcumin and tetrahydrocurcumin induce cell death in Ara-C-resistant acute myeloid leukemia. Phytother Res 33: 1199-1207, 2019.
5. Lee TY and Tseng YH: The potential of phytochemicals in oral cancer prevention and therapy: A review of the evidence. Biomolecules 10: 1150, 2020.

6. Kouhpeikar H, Butler AE, Bamian F, Barreto GE, Majeed M and Sahebkar A: Curcumin as a therapeutic agent in leukemia. J Cell Physiol 234: 12404-12414, 2019.

7. Song X, Zhang M, Dai E and Luo Y: Molecular targets of curcumin in breast cancer (Review). Mol Med Rep 19: 23-29, 2019.

8. Holeček M: Branched-chain amino acids in health and disease: Metabolism, alterations in blood plasma, and as supplements. Nutr Metab (Lond) 15: 33, 2018.

9. Neinast M, Murashige D and Arany Z: Branched chain amino acids. Annu Rev Physiol 81: 139-164, 2019.

10. Mayers JR, Wu C, Clish CB, Kraft P, Torrence ME, Fiske BP, Yuan C, Bao Y, Townsend MK, Tworoger SS, et al: Elevation of circulating branched-chain amino acids is an early event in human pancreatic adenocarcinoma development. Nat Med 20: 1193-1198, 2014.

11. Watanabe A, Higashi T, Sakata T and Nagashima H: Serum amino acid levels in patients with hepatocellular carcinoma. Cancer 54: 1875-1882, 1984.

12. Li Z and Zhang H: Reprogramming of glucose, fatty acid and amino acid metabolism for cancer progression. Cell Mol Life Sci 73: 377-392, 2016.

13. Sun H, Zhou Y, Skaro MF, Wu Y, Qu Z, Mao F, Zhao S and $\mathrm{Xu}$ Y: Metabolic reprogramming in cancer is induced to increase proton production. Cancer Res 80: 1143-1155, 2020.

14. DeBerardinis RJ and Chandel NS: Fundamentals of cancer metabolism. Sci Adv 2: e1600200, 2016.

15. Zeng B, Zhang X, Zhao J, Wei Z, Zhu H, Fu M, Zou D, Feng Y, Luo $\mathrm{H}$ and Lei Y: The role of DNMT1/hsa-miR-124-3p/BCAT1 pathway in regulating growth and invasion of esophageal squamous cell carcinoma. BMC Cancer 19: 609, 2019.

16. Xu Y, Yu W, Yang T, Zhang M, Liang C, Cai X and Shao Q: Overexpression of BCAT1 is a prognostic marker in gastric cancer. Human Pathol 75: 41-46, 2018.

17. Zhang L and Han J: Branched-chain amino acid transaminase 1 (BCAT1) promotes the growth of breast cancer cells through improving mTOR-mediated mitochondrial biogenesis and function. Biochem Biophys Res Commun 486: 224-231, 2017.

18. Zheng YH, Hu WJ, Chen BC, Grahn TH, Zhao YR, Bao HL, Zhu YF and Zhang QY: BCAT1, a key prognostic predictor of hepatocellular carcinoma, promotes cell proliferation and induces chemoresistance to cisplatin. Liver Int 36: 1836-1847, 2016.

19. Hattori A, Tsunoda M, Konuma T, Kobayashi M, Nagy T, Glushka J, Tayyari F, McSkimming D, Kannan N, Tojo A, et al: Cancer progression by reprogrammed BCAA metabolism in myeloid leukaemia. Nature 545: 500-504, 2017.

20. Raffel S, Falcone M, Kneisel N, Hansson J, Wang W, Lutz C, Bullinger L, Poschet G, Nonnenmacher Y, Barnert A, et al: BCAT1 restricts $\alpha \mathrm{KG}$ levels in AML stem cells leading to IDHmut-like DNA hypermethylation. Nature 551: 384-388, 2017.

21. Tönjes M, Barbus S, Park YJ, Wang W, Schlotter M, Lindroth AM, Pleier SV, Bai AHC, Karra D, Piro RM, et al: BCAT1 promotes cell proliferation through amino acid catabolism in gliomas carrying wild-type IDH1. Nat Med 19: 901-908, 2013.

22. Fox DB and Alvarez JV: Epithelial-to-mesenchymal transition activates Bcat 1 expression to promote recurrent tumor growth.bioRxiv: Dec 9, 2020 (Epub ahead of print). doi: 10.1101/2020.12.08.416479.

23. Gu Z, Liu Y, Cai F, Patrick M, Zmajkovic J, Cao H, Zhang Y, Tasdogan A, Chen M, Qi L, et al: Loss of EZH2 reprograms BCAA metabolism to drive leukemic transformation. Cancer Discov 9: 1228-1247, 2019.

24. Chiarini F, Evangelisti C, McCubrey JA and Martelli AM: Current treatment strategies for inhibiting mTOR in cancer. Trends Pharmacol Sci 36: 124-135, 2015.

25. Cargnello M, Tcherkezian J and Roux PP: The expanding role of mTOR in cancer cell growth and proliferation. Mutagenesis 30: 169-176, 2015.

26. Villar VH, Nguyen TL, Delcroix V, Terés S, Bouchecareilh M, Salin B, Bodineau C, Vacher P, Priault M, Soubeyran P and Durán RV: mTORC1 inhibition in cancer cells protects from glutaminolysis-mediated apoptosis during nutrient limitation. Nat Commun 8: 14124, 2017.

27. Tamaddoni A, Mohammadi E, Sedaghat F, Qujeq D and As'Habi A: The anticancer effects of curcumin via targeting the mammalian target of rapamycin complex 1(mTORC1) signaling pathway. Pharmacol Res 156: 104798, 2020. 
28. Tan HK, Moad AI and Tan ML: The mTOR signalling pathway in cancer and the potential mTOR inhibitory activities of natural phytochemicals. Asian Pac J Cancer Prev 15: 6463-6475, 2014

29. Patel SS, Acharya A, Ray RS, Agrawal R, Raghuwanshi R and Jain P: Cellular and molecular mechanisms of curcumin in prevention and treatment of disease. Crit Rev Food Sci Nutr 60 : 887-939, 2020.

30. Pösel C, Möller K, Fröhlich W, Schulz I, Boltze J and Wagner DC: Density gradient centrifugation compromises bone marrow mononuclear cell yield. PLoS One 7: e50293, 2012.

31. Arber DA and Erba HP: Diagnosis and treatment of patients with acute myeloid leukemia with myelodysplasia-related changes (AML-MRC). Am J Clin Pathol 154: 731-741, 2020.

32. Livak KJ and Schmittgen TD: Analysis of relative gene expression data using real-time quantitative PCR and the 2(-Delta Delta C(T)) method. Methods 25: 402-408, 2001.

33. Su PF and Song SQ: Regulation of mTOR by miR-107 to facilitate glioma cell apoptosis and to enhance cisplatin sensitivity. Eur Rev Med Pharmacol Sci 22: 6864-6872, 2018.

34. Murugan AK: mTOR: Role in cancer, metastasis and drug resistance. Semin Cancer Biol 59: 92-111, 2019.

35. Watson JL, Hill R, Lee PW, Giacomantonio CA and Hoskin DW: Curcumin induces apoptosis in HCT-116 human colon cancer cells in a p21-independent manner. Exp Mol Pathol 84: 230-233, 2008.

36. Mishra D, Singh S and Narayan G: Curcumin induces apoptosis in Pre-B acute lymphoblastic leukemia cell lines via PARP-1 cleavage. Asian Pac J Cancer Prev 17: 3865-3869, 2016.

37. Zou Z, Tao T, Li H and Zhu X: mTOR signaling pathway and mTOR inhibitors in cancer: Progress and challenges. Cell Biosci 10: 31, 2020
38. Eden $\mathrm{A}$ and Benvenisty N: Involvement of branched-chain amino acid aminotransferase (Bcat1/Eca39) in apoptosis. FEBS Lett 457: 255-261, 1999.

39. Rozengurt E, Soares HP and Sinnet-Smith J: Suppression of feedback loops mediated by $\mathrm{PI} 3 \mathrm{~K} / \mathrm{mTOR}$ induces multiple overactivation of compensatory pathways: An unintended consequence leading to drug resistance. Mol Cancer Ther 13: 2477-2488, 2014

40. Tian T, Li X and Zhang J: mTOR signaling in cancer and mTOR inhibitors in solid tumor targeting therapy. Int J Mol Sci 20: 755 , 2019.

41. Wang Y, Zhang J, Ren S, Sun D, Huang HY, Wang H, Jin Y, Li F, Zheng C, Yang L, et al: Branched-chain amino acid metabolic reprogramming orchestrates drug resistance to EGFR tyrosine kinase inhibitors. Cell Rep 28: 512-525.e6, 2019.

42. Gan L, Li C, Wang J and Guo X: Curcumin modulates the effect of histone modification on the expression of chemokines by type II alveolar epithelial cells in a rat COPD model. Int J Chron Obstruct Pulmon Dis 11: 2765-2773, 2016.

43. Yan X, Pan B, Lv T, Liu L, Zhu J, Shen W, Huang X and Tian J: Inhibition of histone acetylation by curcumin reduces alcohol-induced fetal cardiac apoptosis. J Biomed Sci 24: 1, 2017.

44. Laribee RN and Weisman R: Nuclear functions of TOR: Impact on transcription and the epigenome. Genes (Basel) 11: 641, 2020.

45. Viscarra JA, Wang Y, Nguyen HP, Choi YG and Sul HS: Histone demethylase JMJD1C is phosphorylated by mTOR to activate de novo lipogenesis. Nat Commun 11: 796, 2020.

(i) (3) This work is licensed under a Creative Commons EY No No Attribution-NonCommercial-NoDerivatives 4.0 International (CC BY-NC-ND 4.0) License. 